\title{
On Levels in Arrangements of Lines, Segments, Planes, and Triangles*
}

\author{
P. K. Agarwal, ${ }^{1}$ B. Aronov, ${ }^{2}$ T. M. Chan, ${ }^{3}$ and M. Sharir ${ }^{4}$ \\ ${ }^{1}$ Department of Computer Science, Box 90129, Duke University, \\ Durham, NC 27708-0129, USA \\ pankaj@cs.duke.edu \\ ${ }^{2}$ Department of Computer and Information Science, Polytechnic University, \\ Brooklyn, NY 11201-3840, USA \\ aronov@ziggy.poly.edu \\ ${ }^{3}$ Department of Mathematics and Computer Science, University of Miami, \\ Coral Gables, FL 33124-4250, USA \\ tchan@cs.miami.edu \\ ${ }^{4}$ School of Mathematical Sciences, Tel Aviv University, \\ Tel Aviv 69978, Israel \\ and \\ Courant Institute of Mathematical Sciences, New York University, \\ New York, NY 10012, USA \\ sharir@math.tau.ac.il \\ Communicated by János Pach
}

\begin{abstract}
We consider the problem of bounding the complexity of the $k$ th level in an arrangement of $n$ curves or surfaces, a problem dual to, and an extension of, the well-known $k$-set problem. Among other results, we prove a new bound, $O\left(n k^{5 / 3}\right)$, on the complexity of the $k$ th level in an arrangement of $n$ planes in $\mathbb{R}^{3}$, or on the number of $k$-sets in a set of $n$ points in three dimensions, and we show that the complexity of the $k$ th level in an arrangement of $n$ line segments in the plane is $O(n \sqrt{k} \alpha(n / k))$, and that the complexity of the $k$ th level in an arrangement of $n$ triangles in 3-space is $O\left(n^{2} k^{5 / 6} \alpha(n / k)\right)$.
\end{abstract}

* The first, second, and fourth authors have been supported by a grant from the US-Israeli Binational Science Foundation. Pankaj Agarwal has also been supported by a National Science Foundation Grant CCR93-01259, by an Army Research Office MURI Grant DAAH04-96-1-0013, by a Sloan fellowship, and by an NYI award and matching funds from the Xerox Corporation. Boris Aronov has also been supported by NSF Grant CCR-92-11541 and a Sloan Research Fellowship. Micha Sharir has also been supported by NSF Grants CCR-94-24398 and CCR-93-11127, and by a grant from the GIF, the German-Israeli Foundation for Scientific Research and Development. A preliminary version containing some of the results of this paper has appeared in Proc. 13th ACM Symp. on Computational Geometry, 1997. 


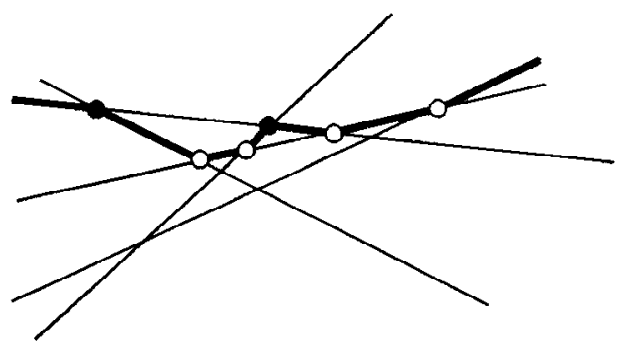

Fig. 1. The third level in an arrangement of lines. The vertices of $V_{2}$ are indicated by empty circles, and the vertices of $V_{3}$ by black circles.

\section{Introduction}

\section{Background}

The $k$-set problem is one of the most challenging open problems in combinatorial geometry. The simplest variant of the problem is: Given a set $S$ of $n$ points in the plane in general position, and a parameter $0 \leq k \leq n-2$, what is the maximum possible number of lines that pass through a pair of points of $S$ and have exactly $k$ points of $S$ in one of the open half-planes that they define ${ }^{1}$ In a dual setting, we are given a set $\mathcal{L}$ of $n$ lines in the plane in general position, and want to bound the maximum possible number of vertices $v$ of the arrangement $\mathcal{A}(\mathcal{L})$, so that exactly $k$ lines pass below $v$. We denote this set of vertices by $V_{k}$. The $k$ th level of the arrangement is defined to be the closure of the set of points that lie on the lines and have exactly $k$ lines below them. The set of vertices of this level is $V_{k} \cup V_{k-1}$. See Fig. 1 for an illustration.

The $k$-set problem was first studied about 1970 by Erdős et al. [14] and Lovász [22]. These papers have established an upper bound $O(n \sqrt{k})$ and a lower bound $\Omega(n \log k)$ on the desired quantity, for $k>0$, leaving a fairly big gap. The upper bound was slightly improved to $O\left(n \sqrt{k} / \log ^{*} k\right)$ by Pach et al. [25]. After the original submission of this paper, significant progress has been made by Dey [8], who improved the upper bound to $O\left(n k^{1 / 3}\right)$, for $k>0$. Dey's proof is based on some of the ideas presented in this paper.

In the dual setting, the problem can be generalized in an obvious manner: In the plane, we are given a collection $\Sigma$ of $n x$-monotone curves, each being the graph of a continuous totally or partially defined function, and a parameter $0 \leq k<n$, and wish to bound the complexity (i.e., the number of vertices) of the $k$ th level in the arrangement $\mathcal{A}(\Sigma)$, defined exactly as in the case of lines. In this more general setting only two results are known: A recent seemingly weak, but elegant analysis by Tamaki and Tokuyama [28] yields the bound $O\left(n^{23 / 12}\right)$ on the complexity of a level in an arrangement of $n$ pseudoparabolas, which are graphs of total functions, each pair of which intersect at most twice. We also mention the case of pseudolines, which in this context are graphs of continuous totally

\footnotetext{
${ }^{1}$ Actually, the $k$-set problem seeks bounds on the number of subsets of $S$ of size $k$ that can be separated from their complements by a line. This quantity and the one defined above are related but not identical (they do have the same asymptotic worst-case behavior); see [2] for a recent survey that discusses this issue.
} 
defined functions, each pair of which intersect exactly once, where a slightly larger lower bound of $\Omega\left(n \cdot 2^{c \sqrt{\log n}}\right)$ for the complexity of the median level is established in [21]. Our proof techniques and upper bounds apply equally well to the case of pseudolines. Recently, Dey's techniques have also been extended to the case of pseudolines [9], [29].

Similar extensions apply in higher dimensions. In the primal setting, we are given a set $S$ of $n$ points in $\mathbb{R}^{d}$ in general position, and wish to bound the number of hyperplanes $\pi$ passing through $d$ of the points such that one of the half-spaces bounded by $\pi$ contains exactly $k$ points of $S{ }^{2}$ For $d=3$, the best-known upper and lower bounds are, respectively, $O\left(n^{8 / 3}\right)$ and $\Omega\left(n^{2} \log n\right)$ [4], [10]. For $d>3$, the best-known upper bound is $O\left(n^{d-c_{d}}\right)$, for some exponentially small but positive constant $c_{d}$ [30]. Note that, in contrast to the planar case, these bounds depend only on $n$ and not on $k$.

We can formulate the problem in an arbitrary dimension, in a dual setting: We consider an arrangement of hyperplanes, or, more generally, of surfaces that are graphs of continuous total or partial functions, and define the $k$ th level of the arrangement exactly as in the planar case. We now seek bounds on the maximum possible number of vertices (or of faces of all dimensions) of the level. Except for the case of hyperplanes, which is equivalent to (a variant of) the $k$-set problem mentioned in the preceding paragraph, no nontrivial bounds for the entire range of values of $k$ are known.

In spite of the sorry state of the problem, even after Dey's improvements, we can obtain improved nontrivial bounds when $k$ is small. The probabilistic analysis of Clarkson and Shor [7] (see also [26]) yields fairly sharp bounds on the combined complexity of the first $k$ levels in arrangements. For the case of hyperplanes, for example, the bound is $\Theta\left(n^{\lfloor d / 2\rfloor} k^{\lceil d / 2\rceil}\right)$. For sufficiently small $k$, this gives a better upper bound on the complexity of a single level than the general bound stated above.

\section{New Results}

In this paper we make several contributions to these problems.

In the preliminary version of this paper [1], we briefly reviewed and simplified some old proofs of the upper bound $O(n \sqrt{k})$ for the original planar $k$-set problem (or, dually, for the case of the $k$ th level in an arrangement of $n$ straight lines in the plane). These proofs are related to the proof technique of Gusfield [16], [17]. We also gave a simple proof of the dual version of what is known as the "Lovász lemma" that is used to prove the bound. These techniques apply equally well to arrangements of pseudolines; see, for example, [15]. For the sake of brevity, this part is not included in this version of the paper.

We adapt our proof techniques to obtain the bound $O\left(n^{3 / 2}\right)$ on the complexity of the $k$ th level in an arrangement of $n$ line segments (or "pseudosegments," to be defined below). The same bound also follows from a result by Katoh et al. [20]. We then describe two simple approaches that reduce the bound to $O(n \sqrt{k} \alpha(n / k))$, where $\alpha(n)$ is the inverse Ackermann function.

\footnotetext{
${ }^{2}$ Again, in the actual $k$-set problem we want to bound the number of subsets of size $k$ that can be separated from their complements by a hyperplane; see [2].
} 
We then proceed to study the $k$-set problem for higher-dimensional point sets (or, dually, though not quite equivalently, to bound the complexity of the $k$ th level in a hyperplane arrangement). In the preliminary version of this paper [1], we have observed that the $O\left(n^{8 / 3}\right)$ bound in $\mathbb{R}^{3}$ can be immediately brought down to $O\left(n^{2} k^{2 / 3}\right)$, if we exploit a simple improved version of the Lovász lemma in an arbitrary dimension derived in this paper. However, using Clarkson and Shor's technique, we improve this bound further to $O\left(n k^{5 / 3}\right)$, in a manner that makes no use of the improved Lovász lemma. Although the improved lemma has so far no significant applications, we include it here because we believe it to be of independent interest and an extension of it is needed for the case of triangles in $\mathbb{R}^{3}$. Over the whole range of $k>0$, the $O\left(n k^{5 / 3}\right)$ bound is stronger than all previous bounds, including the aforementioned $O\left(n k^{2}\right)$ bound on the overall complexity of the first $k$ levels. A similar improved bound, of the form $O\left(n^{\lfloor d / 2\rfloor} k^{\lceil d / 2\rceil-c_{d}}\right)$, can be obtained in any dimension $d>3$, for the same constant $c_{d}>0$ obtained by Živaljević and Vrećica [30]. Again, this bound is the best-known upper bound, for all values of $k>0$.

Finally, we consider the problem of bounding the complexity of the $k$ th level in an arrangement of $n$ triangles in 3-space. We first obtain a nontrivial bound of $O\left(n^{17 / 6}\right)$ and then improve it to $O\left(n^{2} k^{5 / 6} \alpha(n / k)\right)$. Our bound strongly depends on an upper bound on the complexity of a single level in an arrangement of line segments in the plane.

As discussed above, Dey's results supersede some of the bounds obtained in this paper, but they do rely on some of the machinery developed here. Some other, more recent bounds derived here can be further improved by exploiting Dey's results. We have written this revised version of our paper in a manner that is mostly independent of Dey's progress. Nevertheless, for completeness, we conclude the paper with a brief overview of the connections between Dey's results and ours.

\section{Arrangements of Segments}

\subsection{A First Bound}

Let $S$ be a collection of $n$ segments in the plane in general position. For $k=0, \ldots, n-1$, the $k$ th level in the arrangement $\mathcal{A}(S)$ of $S$ is defined to be the closure of the set of all points $w$ that lie on segments of $S$ and are such that the open downward-directed vertical ray emanating from $w$ intersects exactly $k$ segments of $S$ (i.e., there are $k$ segments of $S$ below $w)$. Unlike the case of lines, a level of $\mathcal{A}(S)$ is not necessarily connected. It may involve vertical jumps from a segment to the segment lying directly above or below it, when a new segment starts or ends at a point below the level. The complexity of a level is the number of vertices of $\mathcal{A}(S)$ that lie on the level plus the number of discontinuities of the level. Clearly, the number of such discontinuities is at most $2 n$. We define $V_{k}$, for $k=0, \ldots, n-2$, to be the set of vertices of $\mathcal{A}(S)$ (excluding segment endpoints and points of discontinuity) that have exactly $k$ segments passing below them. The set of vertices of the $k$ th level, excluding segment endpoints and jump discontinuities, is $V_{k-1} \cup V_{k}$. The level bends to the left at vertices of $V_{k-1}$ and to the right at vertices of $V_{k}$. See Fig. 2 for an illustration. 


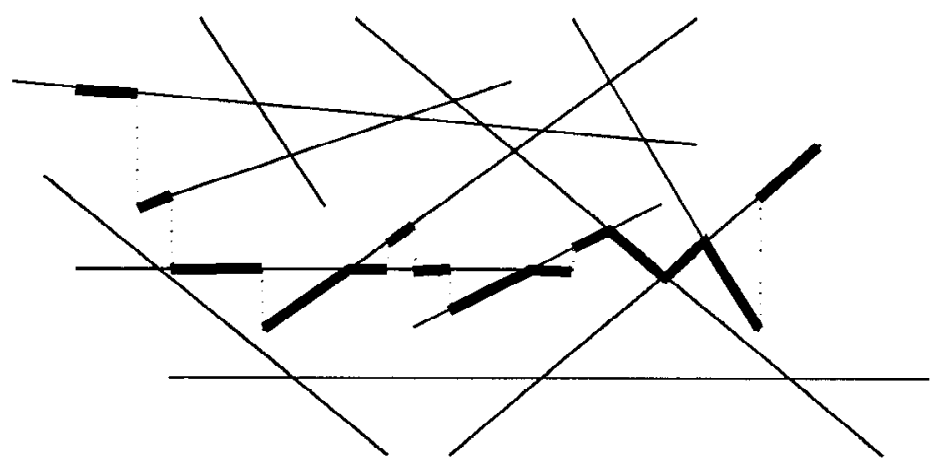

Fig. 2. The second level in an arrangement of segments; here $\left|V_{1}\right|=1$ and $\left|V_{2}\right|=4$.

Theorem 2.1. The complexity of any single level in an arrangement of $n$ line segments in the plane in general position is $O\left(n^{3 / 2}\right)$.

Proof. We first extend to the case of segments the notion of concave chains, introduced in the preliminary version of this paper [1] for arrangements of lines. These chains are not a new concept, and have been used in various earlier works, such as [16] and [17]. The chains are constructed as follows. We start a new chain:

(i) at the left endpoint of any segment (or, if the segment is a line or a leftwarddirected ray, at a point at $x=-\infty$ on this line or ray) if that point lies below the $k$ th level; and

(ii) at any point of discontinuity of the level, when the level jumps up from a segment $s_{i}$ to a segment $s_{j}$ (the chain is started along the lower segment $s_{i}$ ).

As $x$ increases, each chain $c$ follows the segment that it lies on, except when one of the following situations occurs:

(i) $c$ reaches the right endpoint of that segment, and then $c$ terminates there;

(ii) $c$ follows a segment $s_{i}$ and reaches a discontinuity of the $k$ th level, where the level jumps down to $s_{i}$, in which case $c$ is terminated at that point; or

(iii) $c$ reaches a vertex $v \in V_{k-1}$, in which case $c$ bends to the right, and continues along the other segment incident to $v$.

We thus get a collection of at most $2 n$ concave chains (if all the segments are lines, we get exactly $k$ chains). See Fig. 3 for an illustration in the case of lines.

It is easily seen that the resulting chains satisfy the following properties:

(a) The union of the chains is the closure of the portion of the union of the segments that lies below the $k$ th level. Except for the vertices of $V_{k-1}$, the union of the chains lies strictly below the $k$ th level. 


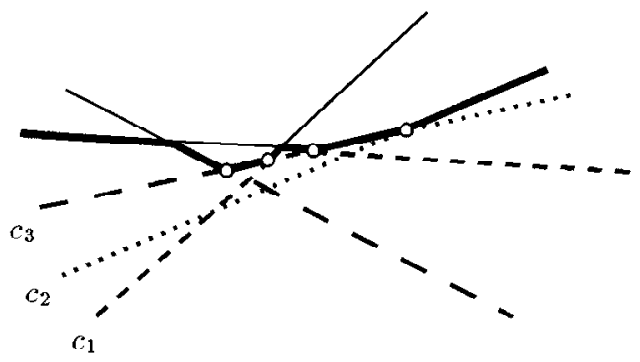

Fig. 3. The concave chains associated with the third level in an arrangement of lines. The level itself is drawn in bold; the dashed paths denote the concave chains $c_{1}, c_{2}, c_{3}$; and the circles denote the vertices of $V_{2}$.

(b) The chains are vertex-disjoint and have nonoverlapping edges, but they may cross each other.

(c) All the vertices of the chains lie on the upper envelope of the chains. Indeed, each chain, except for its vertices, lies fully below the $k$ th level, so any vertex of any chain lies above all the chains that are not incident to it.

Gusfield's analysis [16], [17] can be adapted to obtain the following bound, which clearly implies Theorem 2.1. The same result, for the case of lines, was obtained independently by Halperin and Sharir [18], who were not aware of Gusfield's earlier work.

Theorem 2.2. The overall number of vertices of $t$ concave chains that have nonoverlapping edges (and are thus vertex-disjoint), in an arrangement of $n$ segments in the plane, is $O(n \sqrt{t})$.

Proof. In the preliminary version of this paper [1], the theorem was proved first for the case of lines and then extended to the case of segments. Two proofs were given there, and we give here only one of them; see [1] for more details.

We use the following potential function technique, which is a variant of the technique of [16] and [17]. Let the segments in $S$ be $s_{1}, s_{2}, \ldots, s_{n}$, sorted in the order of decreasing slopes of their containing lines, and let $V$ denote the set of vertices of the chains (excluding chain endpoints). For each $x \in \mathbb{R}$, define

$$
\Phi(x)=\sum\left\{j \mid \ell_{j} \text { lies on one of the chains at } x\right\} .
$$

We clearly have $\Phi(-\infty), \Phi(+\infty)=O(n t)$ (for bounded segments, both quantities are zero; as a matter of fact, $\Phi(x)=O(n t)$ for each $x)$. As we sweep $\mathcal{A}(S)$ with a vertical line from left to right, the value of $\Phi(x)$ can change only when either a chain starts or ends at $x$, or $x$ equals the abscissa $v_{x}$ of a vertex $v \in V$ (refer to Figs. 2 or 3). Suppose that $v \in V$ is the intersection point of segments $s_{i}$ and $s_{j}$, with $j>i$. Then, as easily checked, the change $\Delta \Phi\left(v_{x}\right)=\Phi\left(v_{x}+\varepsilon\right)-\Phi\left(v_{x}-\varepsilon\right)$, for a sufficiently small $\varepsilon>0$, is $j-i>0$. If a chain starts or ends at $x$, then $|\Delta \Phi(x)| \leq n$, and there are at most $2 t$ 


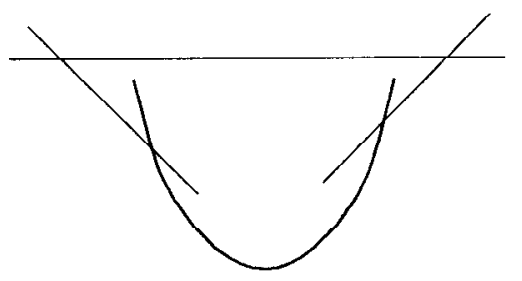

Fig. 4. These four arcs do not form an arrangement of pseudosegments.

such points. In other words,

$$
\sum_{v \in V} \Delta \Phi\left(v_{x}\right)=\Phi(+\infty)-\Phi(-\infty)+O(n t)=O(n t),
$$

with each of these changes being a positive integer.

The number of vertices $v$ at which $\Delta \Phi\left(v_{x}\right)>\sqrt{t}$ is no more than $O(n \sqrt{t})$, as the sum of $\Delta \Phi\left(v_{x}\right)$ at these vertices is $O(n t)$, and each term in the sum is larger than $\sqrt{t}$. The set of vertices $v$ at which the change is at most $\sqrt{t}$ consists of at most $n-1$ vertices with corresponding pairs of indices $(i, i+1), n-2$ vertices with pairs $(i, i+2)$, etc., so its total size is at most

$$
(n-1)+(n-2)+\cdots+(n-\sqrt{t}+1)<n \sqrt{t} .
$$

Combining the two estimates, we conclude that $|V|=O(n \sqrt{t})$.

Remark. The proof of Theorem 2.2 (as well as the other proof given in [1]) also applies to the cases of pseudolines and pseudosegments. We have already defined the notion of a family of pseudolines. A collection $S$ of $n x$-monotone connected arcs is $a$ family of pseudosegments if each of them can be extended to an $x$-monotone connected unbounded curve, so that this family of curves is a collection of pseudolines. (This is a much stronger definition than just requiring each pair of pseudosegments to intersect at most once; see Fig. 4.) We leave it to the reader to verify that the proof goes through in the case of pseudosegments, with straightforward modifications.

\subsection{A k-Sensitive Bound}

Theorem 2.1 does not yield an upper bound for the $k$ th level that depends on $k$. For $k \ll \sqrt{n}$, the known $O(n k \alpha(n / k))$ bound for the overall complexity of the first $k$ levels [26] is actually smaller than the $O\left(n^{3 / 2}\right)$ bound. We offer two approaches for deriving better $k$-sensitive bounds for the $k$ th level, obtaining the following result:

Theorem 2.3. The complexity of the kth level in an arrangement of $n$ line segments in the plane in general position is $O(n \sqrt{k} \alpha(n / k))$, for $k>0$. 
Proof. The first approach involves a bound on the complexity of the $k$ th level in terms of the complexity of higher levels. Recall that the complexity of the $k$ th level, which we will denote by $N_{k}$, is the total number of its (inner) vertices and discontinuous jumps.

Lemma 2.4. $N_{k}=O\left(N_{j} \sqrt{j}\right)$ for any $k<j \leq n$.

Proof. Divide the plane into $O\left(N_{j} / j\right)$ vertical slabs so that each slab contains $O(j)$ vertices/discontinuities of the $j$ th level. Given one such slab $\tau$, let $S_{\tau}$ be the set of segments that contain a point on a level $\leq j$ inside $\tau$. Each segment $s \in S_{\tau}$ is of one of the following three types:

(i) $s$ is on a level $\leq j$ at the left wall of $\tau$.

(ii) $s$ is on a level $>j$ at the left wall. In this case, $s$ must cross the $j$ th level within $\tau$, so it must contain a vertex or a discontinuity on the $j$ th level.

(iii) $s$ does not intersect the left wall of $\tau$. If the left endpoint of $s$ lies below the $j$ th level, then the endpoint causes a discontinuity in the level; otherwise, $s$ crosses the $j$ th level within $\tau$, so the argument for case (ii) applies.

The number of segments in the first case is clearly at most $j+1$. The number of segments in the next two cases is $O(j)$ by our construction of the slabs. We therefore conclude that $\left|S_{\tau}\right|=O(j)$.

As $k<j$, the $k$ th level in $\mathcal{A}(S)$ coincides with the $k$ th level in $\mathcal{A}\left(S_{\tau}\right)$ when restricted to $\tau$. By Theorem 2.1, the complexity of the $k$ th level within each slab is $O\left(j^{3 / 2}\right)$. The total complexity of the $k$ th level in $\mathcal{A}(S)$ is thus $O\left(\left(N_{j} / j\right) \cdot j^{3 / 2}\right)$.

The theorem can now be proved as follows:

$$
N_{k}=O\left(\frac{1}{k} \sum_{k<j \leq 2 k} N_{j} \sqrt{j}\right)=O\left(\frac{1}{\sqrt{k}} \sum_{j \leq 2 k} N_{j}\right)=O(n \sqrt{k} \alpha(n / k))
$$

since the first $2 k$ levels have complexity $O(n k \alpha(n / k))$ [26].

The above proof uses the known upper bound on the combined complexity of the first $O(k)$ levels, which is actually proved using the probabilistic technique of Clarkson and Shor [7]. We now describe a second proof that directly applies Clarkson and Shor's technique.

Take a random sample $R \subseteq S$ of size $r=\lfloor n / 2 k\rfloor$. Let $\mathcal{L} \mathcal{E}(R)$ be the closure of the region beneath the lower envelope (i.e., the 0 th level) in $\mathcal{A}(R)$. By inserting vertical downward-directed rays at each vertex and discontinuity point, we obtain a decomposition of $\mathcal{L} \mathcal{E}(R)$ into "semi-unbounded" trapezoids (the "vertical decomposition"). Each trapezoid is defined by at most three segments, and the number of trapezoids is proportional to the complexity of the lower envelope, which is $\eta(r)=O(r \alpha(r))$ [27].

We now estimate $E[N]$, the expected number of $k$ th level vertices that lie in $\mathcal{L} \mathcal{E}(R)$. For each trapezoid $\tau$, let $S_{\tau}$ be the set of segments of $S$ that intersect $\tau$ (the "conflict list"). Inside $\tau$, the $k$ th level in $\mathcal{A}(S)$ coincides with the $k$ th level in $\mathcal{A}\left(S_{\tau}\right)$ and thus has 
$O\left(\left|S_{\tau}\right|^{3 / 2}\right)$ vertices by Theorem 2.1. It follows that

$$
E[N]=O\left(E\left[\sum_{\tau}\left|S_{\tau}\right|^{3 / 2}\right]\right) .
$$

The standard probabilistic analysis of Clarkson and Shor reveals that this quantity is $O\left(\eta(r) \cdot(n / r)^{3 / 2}\right)=O(n \sqrt{k} \alpha(n / k))$.

On the other hand, for a fixed $k$ th level vertex $v$, the probability that $v \notin \mathcal{L E}(R)$ is the probability that one of the $k$ segments below $v$ is chosen in the random sample $R$; this probability is at most $k r / n \leq 1 / 2$. Therefore, $E[N]$ is at least half the number of $k$ th level vertices, and the second proof is completed.

Remarks. (1) The ideas in both proofs apply to pseudosegments and other families of curves in the plane. For instance, Tamaki and Tokuyama's $O\left(n^{23 / 12}\right)$ bound on the $k$ th level in an arrangement of $n$ pseudoparabolas [28], as mentioned in the Introduction, improves to $O\left(n k^{11 / 12}\right)$ (for the first proof, we can use the known $O(n k)$ bound [26] on the first $O(k)$ levels).

(2) The second proof of the theorem is fairly general, and we will apply the same technique twice more later in this paper (in the proofs of Theorems 3.1 and 5.3). In general, for analyzing the complexity of the $k$ th level in an arrangement of curves or surfaces, we take a random sample of about $n / k$ of the surfaces, compute their lower envelope, and construct the vertical decomposition of the region below the envelope. Within each cell $\tau$ of the decomposition, the $k$ th level of the whole arrangement coincides with the $k$ th level of the subarrangement formed by the surfaces that cross $\tau$. The number of these surfaces is "on the average" only $O(k)$. Roughly speaking, we complete the analysis by applying any insensitive bound on the complexity of the level within each $\tau$, and by multiplying the bound by the number of cells of the decomposition. We hope that this technique will find further applications on top of those obtained in this paper.

\section{Arrangements of Planes}

Let $P=\left\{\pi_{1}, \ldots, \pi_{n}\right\}$ be a collection of $n$ planes in 3-space in general position, and let $\mathcal{A}(P)$ denote the arrangement of $P$. The $k$ th level of $\mathcal{A}(P)$ is defined as the closure of the set of all points that lie in the union of the planes and have exactly $k$ planes lying below them. The complexity of the level, regarded as a polyhedral surface, is the number of its vertices, edges, and faces. This is clearly proportional to only the number of vertices, and we will focus on bounding this latter quantity.

Theorem 3.1. The number of vertices of the kth level of $\mathcal{A}(P)$ is $O\left(n k^{5 / 3}\right)$, for $k>0$.

This theorem improves the bound $O\left(n^{8 / 3}\right)$ established in [10] (see also [3]) for $k \ll n$, and is also better than the bound $O\left(n k^{2}\right)$ on the overall complexity of the first $k$ levels.

Proof. We follow the second proof of Theorem 2.3 and consider a random sample $R \subseteq P$ of size $r=\lfloor n / 2 k\rfloor$. The analysis by Clarkson and Shor [7] implies that $\mathcal{L} \mathcal{E}(R)$, 
the region beneath the lower envelope of $R$, can be decomposed into $O(r)$ vertical triangular prisms $\left\{\tau_{i}\right\}$ so that each prism is defined by a constant number of planes, and

$$
E\left[\sum_{i}\left|P_{\tau_{i}}\right|^{8 / 3}\right]=O\left(r \cdot(n / r)^{8 / 3}\right)=O\left(n k^{5 / 3}\right),
$$

where $P_{\tau_{i}}$ denotes the set of planes of $P$ intersecting $\tau_{i}$. Inside each prism $\tau_{i}$, the $k$ th level of $\mathcal{A}(P)$ coincides with the $k$ th level of $\mathcal{A}\left(P_{\tau_{i}}\right)$, because any plane in $P \backslash P_{\tau_{i}}$ passes above $\tau_{i}$. Hence, the number of $k$ th level vertices (of $\mathcal{A}(P)$ ) inside each $\tau_{i}$ is $O\left(\left|P_{\tau_{i}}\right|^{8 / 3}\right.$ ) by Dey and Edelsbrunner's bound [10]. The expected number, $E[N]$, of $k$ th level vertices inside $\mathcal{L} \mathcal{E}(R)$, is thus $O\left(n k^{5 / 3}\right)$ by (1). The proof is completed by observing, as in the proof of Theorem 2.3, that $E[N]$ is at least half the total number of $k$ th level vertices.

Instead of the above probabilistic argument, an alternative proof can be obtained from results on geometric cuttings, specifically, the shallow cuttings of Matoušek [23]:

Let $H$ be a collection of $n$ hyperplanes in $\mathbb{R}^{d}$ in general position. A $(1 / r)$-cutting of the first $k$ levels is a collection of simplices $\left\{\tau_{i}\right\}$ covering all points of levels $\leq k$, such that $\left|H_{\tau_{i}}\right| \leq n / r$ for each $i$, where $H_{\tau_{i}}$ is the set of all hyperplanes of $H$ that intersect the interior of $\tau_{i}$.

Lemma 3.2 (Matoušek's Shallow Cutting Lemma). Let $r \leq n$ and $q=k(r / n)+1$. There exists a $(1 / r)$-cutting for the first $k$ levels of $\mathcal{A}(H)$, consisting of $O\left(r^{\lfloor d / 2\rfloor} q^{\lceil d / 2\rceil}\right)$ simplices.

Second Proof of Theorem 3.1. We can prove our theorem by setting $d=3, r=n / k$, and $q=2$ in the above lemma. We obtain a collection of $O(n / k)$ simplices, each of which is intersected by $O(k)$ planes and consequently contains $O\left(k^{8 / 3}\right)$ vertices of the $k$ th level of $\mathcal{A}(P)$. (This follows from the fact that the intersection of the $k$ th level with a simplex $\tau_{i}$ lies at a fixed level $(\leq k)$ of $\mathcal{A}\left(P_{\tau_{i}}\right)$, where, as above, $P_{\tau_{i}}$ is the set of planes crossing $\tau_{i}$.) Hence, the total number of $k$ th level vertices is $O\left((n / k) \cdot k^{8 / 3}\right)=O\left(n k^{5 / 3}\right)$. However, we prefer the earlier proof, as the proof of the shallow cutting lemma itself requires actually more involved probabilistic techniques, including an argument similar to one of the previous proofs [23].

Remark. As before, any improvement in the worst-case $k$-insensitive upper bound would imply an improvement in our $k$-sensitive bound. Extensions to $d$-dimensional arrangements of hyperplanes are also immediate: if we have an $O\left(n^{d-c_{d}}\right)$ bound on the complexity of a single level, then the complexity of the $k$ th level is $O\left(r^{\lfloor d / 2\rfloor} \cdot(n / r)^{d-c_{d}}\right)=$ $O\left(n^{\lfloor d / 2\rfloor} k^{\lceil d / 2\rceil-c_{d}}\right)$, as the number of simplices used in either proof is $O\left(r^{\lfloor d / 2\rfloor}\right)$.

\section{Improved Lovász Lemma in Higher Dimensions}

In the preliminary version of this paper [1], we have obtained the $k$-sensitive bound of $O\left(n^{2} k^{2 / 3}\right)$, which is weaker than the one in Theorem 3.1. The proof follows the techniques of [3] and [10], which exploit a generalization of the Lovász lemma to three 
dimensions. We present here an improved version of this lemma, in arbitrary dimension, that has a simple proof and may be of independent interest. So far, the improved lemma has no significant applications because the best upper bounds, derived above, are not based on it. We, however, believe that the lemma deserves exposition because it has been one of the very few tools for attacking the $k$-set problem so far and a generalization of the lemma is needed in Section 5.

Let $H$ be a collection of $n$ hyperplanes in $\mathbb{R}^{d}$ in general position, and let $0 \leq k \leq n-d$. Let $V_{k}$ denote the set of those vertices $v$ of $\mathcal{A}(H)$ for which exactly $k$ hyperplanes of $H$ pass below $v$. For each $v \in V_{k}$, we denote by $H_{v}$ the set of the $d$ hyperplanes incident to $v$, and let $R_{v}$ denote the closed region ("corridor") lying between the upper and lower envelopes of the hyperplanes of $H_{v}$.

Lemma 4.1 (Dual Lovász Lemma in Arbitrary Dimension). For any $(d-2)$-flat $f$ in $\mathbb{R}^{d}$, we have

$$
\left|\left\{v \in V_{k} \mid f \subset R_{v}\right\}\right|=O\left(k^{d-1}\right) .
$$

The previous bound was $O\left(n^{d-1}\right)$ (see [4] and [22]). It will be more convenient to state and prove the primal version of this lemma. Fix a set $S$ of $n$ points in $\mathbb{R}^{d}$, in general position. A $k$-facet is a $(d-1)$-dimensional simplex spanned by $d$ points of $S$ with the property that its affine hull has precisely $k$ points of $S$ on one side of it.

Lemma 4.2 (Primal Lovász Lemma in Arbitrary Dimension). Let $S$ be a finite point set in $\mathbb{R}^{d}$. Then, for any line $\ell$, the number of $k$-facets meeting $\ell$ is $O\left(k^{d-1}\right)$.

Proof. Note that this formulation of the lemma is independent of the choice of the coordinate system. Construct a coordinate system in which $\ell$ coincides with the $x_{d^{-}}$ axis. Dualize $S$ to a system $S^{*}$ of $n$ hyperplanes, using the standard duality that maps a point $\left(a_{1}, \ldots, a_{d}\right)$ to the hyperplane $x_{d}=-a_{1} x_{1}-a_{2} x_{2}-\cdots-a_{d-1} x_{d-1}+a_{d}$, and a hyperplane $x_{d}=b_{1} x_{1}+b_{2} x_{2}+\cdots+b_{d-1} x_{d-1}+b_{d}$ to the point $\left(b_{1}, \ldots, b_{d}\right)$ (see, e.g., [11]); this duality preserves incidences and above-below relationships between points and hyperplanes (i.e., a point $p$ lies below, on, or above a hyperplane $h$ if and only if the dual hyperplane $p^{*}$ of $p$ lies below, on, or above the point $h^{*}$ dual to $h$ ). An application of such a duality also shows that this lemma and the preceding one are indeed dual versions of each other. It suffices to count the number of $k$-facets whose affine hulls have $k$ points of $S$ strictly below them. The remaining class of $k$-facets is handled by a symmetric argument.

The properties of the duality imply that the affine hull of a $k$-facet $\Delta$ as above is mapped into a vertex $\Delta^{*}$ of the arrangement of $S^{*}$ that has precisely $k$ hyperplanes below it (and $d$ hyperplanes passing through it). Hence $\Delta^{*}$ is a vertex of the $k$ th level of $\mathcal{A}\left(S^{*}\right)$. Moreover, $\Delta$ meets the $x_{d}$-axis $\ell$ if and only if the horizontal hyperplane through $\Delta^{*}$ is contained in $R_{\Delta^{*}}$, i.e., $\Delta^{*}$ is a local maximum of the $k$ th level of $\mathcal{A}\left(S^{*}\right){ }^{3}$ Indeed, $\Delta$

\footnotetext{
3 The connection between the local extrema of $k$ th levels and the Lovász lemma was first observed by Clarkson, as briefly remarked in the Introduction of [5].
} 
meets $\ell$ if and only if every hyperplane that contains $\ell$ does not have all vertices of $\Delta$ on one side. The set of these hyperplanes is mapped by our duality to the set of all the points at infinity in horizontal directions. Hence $\Delta$ meets $\ell$ if and only if every point at infinity in a horizontal direction lies in $R_{\Delta^{*}}$, which is equivalent to the condition that the horizontal hyperplane through $\Delta^{*}$ is contained in $R_{\Delta^{*}}$, as asserted. As shown by Clarkson [5], the number of local extrema of the $k$ th level in an arrangement of hyperplanes in $d$-space is $O\left(k^{d-1}\right)$, and this completes the proof of the lemma.

\section{Arrangements of Triangles}

Let $\mathcal{T}=\left\{\Delta_{1}, \ldots, \Delta_{n}\right\}$ be a collection of $n$ triangles in 3-space in general position, and let $\mathcal{A}(\mathcal{T})$ denote the arrangement of $\mathcal{T}$. The $k$ th level of $\mathcal{A}(\mathcal{T})$ is defined, again, as the closure of the set of all points that lie in the union of the triangles and have exactly $k$ triangles below them (i.e., the relatively open vertical downward-directed ray emerging from such a point intersects exactly $k$ triangles). As in the case of segments, the $k$ th level is not necessarily connected, and may have jump discontinuities at points that lie vertically above or on some triangle edge. The complexity of the level, regarded as a polyhedral surface, is the number of its vertices, edges, and faces. Assuming general position, this is clearly proportional to the number of vertices only, and we will focus on bounding the number of inner vertices, which are contained in the interiors of three distinct triangles. Any other "outer" vertex of the level lies in the vertical plane $H_{e}$ spanned by some triangle edge $e$. Moreover, if we intersect all the triangles with $H_{e}$, we get a collection of at most $n$ segments, and the vertices of the $k$ th level of $\mathcal{A}(\mathcal{T})$ that lie in $H_{e}$ are vertices of the $k$ th level of any of the two-dimensional arrangements of these segments within $H_{e}$, where $e$ itself is either included or excluded. By Theorem 2.1, the number of such vertices is $O\left(n^{3 / 2}\right)$. Repeating this analysis for each triangle edge $e$, we conclude that the number of outer vertices of the level is $O\left(n^{5 / 2}\right)$.

We bound the number of inner vertices using a variant of the dual version of the Lovász lemma in 3-space. The bound that we obtain is considerably weaker than the one given in Lemma 4.1, but is still nontrivial. The proof of this version of the lemma is also different and somewhat more involved.

Let $v$ be an inner vertex of the $k$ th level, incident to three triangles $\Delta_{1}, \Delta_{2}, \Delta_{3} ; v$ can be classified into three categories, depending on whether the $k$ th level in the neighborhood of $v$ coincides with

(a) the lower envelope of $\Delta_{1}, \Delta_{2}, \Delta_{3}$;

(b) the first level of the arrangement $\mathcal{A}\left(\left\{\Delta_{1}, \Delta_{2}, \Delta_{3}\right\}\right)$; or

(c) the upper envelope of $\Delta_{1}, \Delta_{2}, \Delta_{3}$.

Note that vertices of type (b) have the property that all six edges of $\mathcal{A}(\mathcal{T})$ incident to the vertex lie on the $k$ th level, whereas for vertices of type (a) or (c), only three of these edges lie on the level, one edge on each segment of intersection of two of the triangles $\Delta_{1}, \Delta_{2}, \Delta_{3}$.

For each inner vertex $v$ of the $k$ th level of type (a) or (c), let $R_{v}$ be the closed region enclosed between the upper envelope and the lower envelope of the three planes 


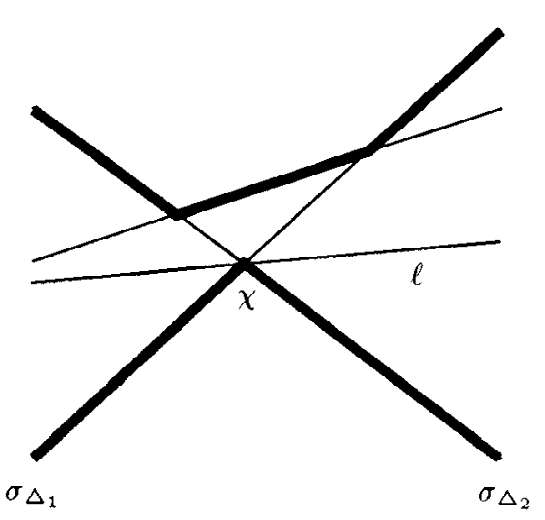

(a)

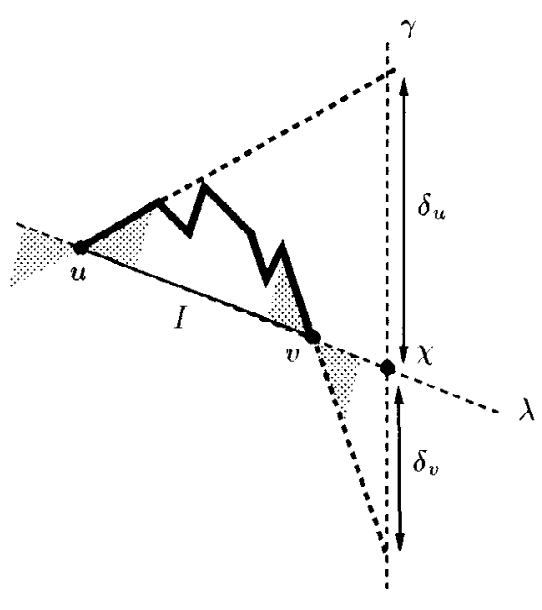

(b)

Fig. 5. (a) Cross-section of a region $R_{v}$ in $H$; the line $\ell$ just becomes contained in $R_{v}$; (b) cross-section of $\mathcal{A}(\mathcal{T})$ by $\pi$; the intersections of $R_{v}, R_{u}$ with $\pi$ are shaded near the respective vertices.

containing the three triangles incident to $v$; see Fig. 5 for a cross-section of such an $R_{v}$. We have the following weaker version of the Lovász lemma:

Lemma 5.1. Any line in $\mathbb{R}^{3}$ is fully contained in at most $O\left(n^{5 / 2}\right)$ regions $R_{v}$ of vertices of types (a) and (c).

Proof. Let $\ell_{1}$ be a line in $\mathbb{R}^{3}$, and let $H$ be the vertical plane containing $\ell_{1}$. For a triangle $\Delta \in \mathcal{T}$, let $\pi_{\Delta}$ be the plane containing $\triangle$ and $\sigma_{\Delta}=\pi_{\Delta} \cap H$. Let $\mathcal{A}_{H}$ be the arrangement in $H$ of the lines $\left\{\sigma_{\Delta} \mid \triangle \in \mathcal{T}\right\}$. Let $\ell_{0}$ be a line contained in $H$, parallel to $\ell_{1}$, and lying below all vertices of $\mathcal{A}_{H}$. It is easily checked that no region $R_{v}$ contains $\ell_{0}$. We will move a line $\ell$ within $H$ upward, parallel to itself, from the position when it coincides with $\ell_{0}$ until it coincides with $\ell_{1}$. We estimate the change in the number of regions $R_{v}$ that contain $\ell$ as it moves. Summing these changes yields the bound on the desired quantity for $\ell_{1}$.

The set of regions $R_{v}$ that fully contain $\ell$ can change only when $\ell$ passes through a vertex of $\mathcal{A}_{H}$. Clearly, the vertex $\chi=\sigma_{\Delta_{1}} \cap \sigma_{\Delta_{2}}$ has to be such that there is an inner type (a) or type (c) vertex $v$ in $\mathcal{A}$ incident to $\Delta_{1}$ and $\Delta_{2}$. Under these assumptions, for $\ell$ to become newly contained in a region $R_{v}$, or to stop being contained in $R_{v}$, as it sweeps past such a vertex $\chi$, it is necessary and sufficient that the slope of $\ell$ lies between the slopes of $\sigma_{\triangle_{1}}$ and $\sigma_{\Delta_{2}}$; see Fig. 5(a). Let $\chi$ be such a vertex (where this latter condition also holds). Put $\lambda=\pi_{\Delta_{1}} \cap \pi_{\triangle_{2}}$. Let $s \subset \lambda$ be the segment $\triangle_{1} \cap \triangle_{2}$. For all regions $R_{v}$ that either start or stop containing $\ell$ as it sweeps over $\chi, v$ is contained in $s$, so it suffices to concentrate only on such regions $R_{v}$.

We mark on $s$ all the inner vertices of the $k$ th level of $\mathcal{A}(\mathcal{T})$ of types (a) and (c), and consider the set of maximal subintervals of $s$ not contained in the $k$ th level. Each such subinterval $I$ is delimited by two points $u, v$, each of which is an inner vertex of the 
level of type (a) or (c) (it cannot be a vertex of type (b) because all six edges incident to a type (b) vertex lie on the $k$ th level), a point of jump discontinuity of the level, or an endpoint of $s$. Let $q_{s}$ be the number of jump discontinuities of the $k$ th level along $s$. Note that each such discontinuity is an outer vertex of the $k$ th level. If an inner vertex $v$ is an endpoint of an interval along $s$ whose other endpoint $v^{\prime}$ is either a jump discontinuity or an endpoint of $s$, we change $v$ to $v^{\prime}$. The number of such inner vertices (and therefore the change in the number of regions that contain $\ell$, corresponding to such vertices) is at most $q_{s}+2$.

Next, consider an interval $I$, both of whose endpoints are inner vertices, say $u$ and $v$. Consider the vertical plane $\pi$ containing $\lambda$, and the cross-section of $\mathcal{A}(\mathcal{T})$ within $\pi$ (refer to Fig. 5(b)). Clearly, the $k$ th level of this cross-section is contained in the $k$ th level of $\mathcal{A}(\mathcal{T})$, so it either lies fully above $I$ or fully below $I$. In the former case both $u$ and $v$ are of type (c), and in the latter case they are both of type (a). Let $\gamma$ be the vertical line $H \cap \pi$, and let $\delta_{u}=R_{u} \cap \gamma$ and $\delta_{v}=R_{v} \cap \gamma$. If $\chi \notin I$, then it is easily checked that $\delta_{u}$ and $\delta_{v}$ lie on opposite sides of $\chi$ along $\gamma$ and thus are disjoint except at their common endpoint $\chi$. This fact, and our assumptions that the slope of $\ell$ is between the slopes of $\sigma_{u}=\triangle_{u} \cap H$ and $\sigma_{v}=\triangle_{v} \cap H$, imply that one of $R_{u}, R_{v}$ must be added, and the other one removed, from the set of regions containing $f$, as $\ell$ sweeps over $\chi$. Hence, as $\ell$ sweeps over $\chi, R_{u}$ and $R_{v}$ "cancel" out each other, in terms of containment of $\ell$.

To summarize, we have shown that as $\ell$ passes through $\chi$, the change in the number of regions $R_{v}$ containing $\ell$ is at most $4+q_{s}$. This implies that the number of regions $R_{v}$ that contain $\ell$ in its final position $\ell_{1}$ is at most $\sum_{s}\left(4+q_{s}\right)$, where the sum is over all $O\left(n^{2}\right)$ intersection segments between pairs of triangles in $\mathcal{T}$. Since the number of outer vertices on the $k$-th level is $O\left(n^{5 / 2}\right)$, as argued above, and each is counted at most three times, $\sum_{s} q_{s}=O\left(n^{5 / 2}\right)$. The number of regions containing $\ell$ is thus $O\left(n^{2}\right)+O\left(n^{5 / 2}\right)=O\left(n^{5 / 2}\right)$, as asserted.

What if $\ell_{1}$ actually passes through a vertex $\chi=\sigma_{\Delta_{i}} \cap \sigma_{\Delta_{j}}$ of $\mathcal{A}_{H}$ ? Then the cancellation does not occur, which adds fewer than $n$ regions $R_{v}$ that can contain $\ell$ - each such region corresponds to some vertex of $\mathcal{A}(\mathcal{T})$ on the segment $\triangle_{i} \cap \triangle_{j}$.

Theorem 5.2. The complexity of any single level in an arrangement of $n$ triangles in 3-space is $O\left(n^{17 / 6}\right)$.

Proof. Lemma 5.1 implies that no line $\ell$ is contained in more than $O\left(n^{5 / 2}\right)$ regions $R_{v}$. Passing to the dual space, we obtain the following equivalent formulation, similar to the case of planes: The planes containing the triangles in $\mathcal{T}$ are mapped to a set of $n$ points. Each inner vertex $v$ of the $k$ th level is mapped to a triangle spanned by the three points dual to the planes containing the triangles incident to $v$. The line $\ell$ is mapped to another line $\ell^{*}$, and $\ell$ is contained in $R_{v}$ if and only if $\ell^{*}$ crosses the triangle dual to $v$. We now have a system of $X$ triangles in 3-space, spanned by a total of $n$ points, where $X$ is the number of inner vertices of the $k$ th level of types (a) and (c). By the result of [10], there exists a line that crosses at least $\Omega\left(X^{3} / n^{6}\right)$ such triangles. On the other hand, by Lemma 5.1, this number is at most $O\left(n^{5 / 2}\right)$. Combining these two inequalities yields $X=O\left(n^{17 / 6}\right)$. We still need to bound the number of vertices of type (b). However, these vertices are vertices of type (a) of the $(k-1)$ st level, so, repeating the above analysis for this level, we obtain the bound asserted in the theorem. 
Theorem 5.3. The complexity of the $k$ th level in an arrangement of $n$ triangles in 3-space is $O\left(n^{2} k^{5 / 6} \alpha(n / k)\right)$, for $k>0$.

Proof. Take a random sample $R \subseteq \mathcal{T}$ of size $r=\lfloor n / 2 k\rfloor$. The result of Pach and Sharir [24] (see also [12] and [27]) implies that the region beneath the lower envelope of $R$ can be decomposed into $\eta(r)=O\left(r^{2} \alpha(r)\right)$ vertical triangular prisms $\left\{\tau_{i}\right\}$, each defined by a constant number of triangles of $\mathcal{T}$. Clarkson and Shor's analysis [7] can be applied to show that

$$
E\left[\sum_{i}\left|\mathcal{T}_{\tau_{i}}\right|^{17 / 6}\right]=O\left(\eta(r) \cdot(n / r)^{17 / 6}\right)=O\left(n^{2} k^{5 / 6} \alpha(n / k)\right),
$$

where $\mathcal{T}_{\tau_{i}}$ denotes the set of triangles of $\mathcal{T}$ intersecting $\tau_{i}$. The rest of the proof now proceeds as in the second proof of Theorem 2.3 or the first proof of Theorem 3.1.

Remark. An open problem is to extend Lemmas 4.1 and 5.1 to the respective cases of pseudohyperplanes and pseudotriangles, under appropriate definitions of these objects, and then to extend the proofs of Theorems 5.2 and 5.3 to these cases. Note that there are two different problems to address: One is to extend the Lovász lemma, and the other calls for a dual and more general version of the analysis technique of [10] (that yields a line that stabs many triangles).

\section{Dey's Improvements}

As promised, we conclude this paper with a brief discussion of Dey's recent results and their interconnections to the results of this paper; we refer to Dey's paper [8] for more details.

\section{The Case of Lines}

Dey's proof for the case of lines (the standard planar $k$-set problem) uses the concave chain structure developed in this paper. His proof essentially shows that the complexity of $k$ concave (unbounded) chains in an arrangement of $n$ lines, which have no overlapping edges, is $O\left(n k^{1 / 3}\right)$. His original proof caters only for the case where the chains are $x$ monotone and unbounded, but a slightly refined argument shows that the complexity of any $k$ convex chains (or convex polygons) in an arrangement of $n$ lines, which do not have overlapping edges, is $O\left(n k^{1 / 3}\right)$ for $k=O(n)$ and $O\left(n^{2 / 3} k^{2 / 3}\right)$ for larger values of $k$. Both bounds can be shown to be tight in the worst case: A matching lower bound for the former case is given by Eppstein [13], and a matching lower bound for the latter case is immediate from the known tight bound on the maximum complexity of $k$ faces in an arrangement of $n$ lines [6]. Earlier work [18], [19] has established the bound $O\left(k^{2 / 3} n^{2 / 3}+n\right)$ for the restricted case in which the concave chains are not allowed to cross each other; in this restricted case, the bound also holds for $k=O(n)$. 


\section{The Case of Segments}

Dey has improved the bound in Theorem 2.1 to $O\left(n^{4 / 3}\right)$. This is an immediate consequence of the extension of his result concerning the complexity of an arbitrary collection of convex chains with nonoverlapping edges in an arrangement of lines (or of segments), as just mentioned.

Concerning the $k$-sensitive bound (Theorem 2.3), it follows from our proofs that any improvement on the worst-case bound over all $k$ leads to an improvement on the $k$-sensitive bound. Thus, combining our analysis with Dey's $O\left(n^{4 / 3}\right)$ bound implies a new bound of $O\left(n k^{1 / 3} \alpha(n / k)\right)$ for the complexity of the $k$ th level in an arrangement of segments.

\section{The Case of Pseudolines and Pseudosegments}

Following the recent results of [9] and [29], Dey's technique can also be extended to the cases of pseudolines and pseudosegments (in the sense defined above). Thus all the bounds just stated also apply to the corresponding cases of pseudolines or of pseudosegments.

\section{The Case of Triangles in 3-Space}

The analysis of the case of triangles relies strongly on bounds for the complexity of a level in a planar arrangement of segments. Thus Dey's results can be "plugged into" the analysis, and improve the bounds in Theorems 5.2 and 5.3 to $O\left(n^{25 / 9}\right)$ and $O\left(n^{2} k^{7 / 9} \alpha(n / k)\right)$, respectively, as is easily checked.

\section{Acknowledgments}

We wish to thank János Pach and Emo Welzl for useful discussions concerning these problems, and Ken Clarkson for pointing out a much simpler reduction from the number of local extrema of $k$-levels to the Lovász lemma, as presented in this paper. We also thank Tamal Dey for communicating his results to us and for useful discussions concerning them.

\section{References}

1. P. K. Agarwal, B. Aronov, and M. Sharir, On levels in arrangements of lines, segments, planes, and triangles, Proc. 13th ACM Symp. on Computational Geometry, 1997, pp. 30-38.

2. A. Andrzejak and E. Welzl, $k$-Sets and $j$-Facets: A survey. Manuscript. In preparation.

3. B. Aronov, B. Chazelle, H. Edelsbrunner, L. Guibas, M. Sharir, and R. Wenger, Points and triangles in the plane and halving planes in space, Discrete Comput. Geom., 6 (1991), 435-442.

4. I. Bárány, Z. Füredi, and L. Lovász, On the number of halving planes, Combinatorica, 10 (1990), 175-183. 
5. K. Clarkson, A bound on local minima of arrangements that implies the Upper Bound Theorem, Discrete Comput. Geom., 10 (1993), 427-433.

6. K. Clarkson, H. Edelsbrunner, L. Guibas, M. Sharir, and E. Welzl, Combinatorial complexity bounds for arrangements of curves and spheres, Discrete Comput. Geom., 5 (1990), 99-160.

7. K. Clarkson and P. Shor, Applications of random sampling in computational geometry II, Discrete Comput. Geom., 4 (1989), 387-421.

8. T. Dey, Improved bounds for planer $k$-sets and related problems, Discrete Comput. Geom., this issue, pp. 373-382. Also in Proc. 38th IEEE Symp. on Foundations of Computer Science, 1997, pp. 156-161.

9. T. Dey, personal communication.

10. T. Dey and H. Edelsbrunner, Counting triangle crossings and halving planes, Discrete Comput. Geom., 12 (1994), 281-289.

11. H. Edelsbrunner, Algorithms in Combinatorial Geometry, Springer-Verlag, Heidelberg, 1987.

12. H. Edelsbrunner, L. Guibas, and M. Sharir, The upper envelope of piecewise linear functions: Algorithms and applications, Discrete Comput. Geom., 4 (1989), 311-336.

13. D. Eppstein, Geometric lower bounds for parametric matroid optimization, Proc. 27th ACM Symp. on Theory of Computing, 1995, pp. 662-671.

14. P. Erdős, L. Lovász, A. Simmons, and E. G. Straus, Dissection graphs of planar point sets, In: A Survey of Combinatorial Theory (J. N. Srivastava et al., eds), North-Holland, Amsterdam, 1973, pp. 139-149.

15. J. E. Goodman and R. Pollack, Allowable sequences and order types in discrete and computational geometry, In: New Trends in Discrete and Computational Geometry, Algorithms and Combinatorics (J. Pach, Ed.), vol. 10, Springer-Verlag, New York, 1993, pp. 103-134.

16. D. Gusfield, Sensitivity Analysis for Combinatorial Optimization, Ph.D. thesis, University of California at Berkeley, 1980.

17. D. Gusfield, Bounds for the parametric minimum spanning tree problem, In: Proc. Humboldt Conference on Graph Theory, Combinatorics and Computing, 1979, Utilitas Mathematica, pp. 173-183.

18. D. Halperin and M. Sharir, On disjoint concave chains in arrangements of (pseudo) lines, Inform. Process. Lett., 40 (1991), 189-192.

19. D. Halperin and M. Sharir, Corrigendum: On disjoint concave chains in arrangements of (pseudo) lines, Inform. Process. Lett., 51 (1994), 53-56.

20. N. Katoh, T. Tokuyama, and K. Iwano, On minimum and maximum spanning trees of linearly moving points, Discrete Comput. Geom., 13 (1995), 161-176.

21. M. Klawe, M. Paterson, and N. Pippenger, unpublished manuscript.

22. L. Lovász, On the number of halving lines, Ann. Univ. Sci. Budapest, Eötvös, Sec. Math., 14 (1971), 107-108.

23. J. Matoušek, Reporting points in halfspaces, Comput. Geom. Theory Appl., 2 (1992), 169-186.

24. J. Pach and M. Sharir, The upper envelope of piecewise linear functions and the boundary of a region enclosed by convex plates: Combinatorial analysis, Discrete Comput. Geom., 4 (1989), 291-309.

25. J. Pach, W. Steiger, and M. Szemerédi, An upper bound on the number of planar $k$-sets, Discrete Comput. Geom., 7 (1992), 109-123.

26. M. Sharir, On $k$-sets in arrangements of curves and surfaces, Discrete Comput. Geom., 6 (1991), 593-613.

27. M. Sharir and P. K. Agarwal, Davenport-Schinzel Sequences and Their Geometric Applications, Cambridge University Press, New York, 1995.

28. H. Tamaki and T. Tokuyama, How to cut pseudo-parabolas into segments, In: Proc. 11th ACM Symp. on Computational Geometry, 1995, pp. 230-237.

29. H. Tamaki and T. Tokuyama, A characterization of planar graphs by pseudo-line arrangements, Manuscript, 1997.

30. R. Živaljević and S. Vrećica, The colored Tverberg's problem and complexes of injective functions, J. Combin. Theory Ser. A, 61 (1992), 309-318.

Received February 7, 1997, and in revised form May 15, 1997, and August 30, 1997. 